\title{
Improving confidence set estimation when parameters are weakly identified
}

\author{
Heather Battey* $^{*} \quad$ Qiang Feng ${ }^{\dagger} \quad$ Richard J. Smith ${ }^{\ddagger}$
}

June 20, 2016

\begin{abstract}
We consider inference in weakly identified moment condition models when additional partially identifying moment inequality constraints are available. We detail the limiting distribution of the estimation criterion function and consequently propose a confidence set estimator for the true parameter.
\end{abstract}

\section{Introduction}

This paper considers the estimation of a $d_{\theta}$-vector of parameters $\theta_{0}$ which is the solution to the set of moment equality restrictions

$$
\mathbb{E}[g(Z, \theta)]=0 \text { at } \theta=\theta_{0}
$$

where $g(z, \theta)$ is a $d_{g}$-vector of known functions of the observation vector $z$ and $\theta \in \Theta$ with $\Theta$ the parameter space. Estimators based on estimating equations of the form (1.1) are referred to as Z-estimators (e.g. van der Vaart, 1998) and have found application in numerous fields, e.g., survival modelling with incomplete covariate data (Lipsitz and Ibrahim, 1998) and causal inference with instrumental variables (Shpitser, 2014; Imbens, 2014).

A challenging problem arises when the identifying strength of the moment conditions (1.1) for $\theta_{0}$ is weak, e.g., when instrumental variables used to construct the moment indicator $g(Z, \theta)$ are only weakly correlated with endogenous covariates. Existing inferential procedures robust to weak identification, see inter alia Stock and Wright (2000), Kleibergen (2005), Guggenberger and Smith (2005), Andrews and Cheng (2012), share the shortcoming that confidence set estimators for $\theta_{0}$ are frequently too large to be of practical use. In many applications where weak identification is a problem, however, moment inequality conditions of the form

$$
\mathbb{E}[m(Z, \theta)] \geq 0 \text { at } \theta=\theta_{0}
$$

\footnotetext{
*Imperial College London and Princeton University. Email: h.battey@imperial.ac.uk.

${ }^{\dagger}$ UIBE, Beijing. Email: q.feng@uibe.edu.cn.

${ }^{\ddagger}$ University of Cambridge. Email: rjs27@econ.cam.ac.uk.
} 
are often available, where $m(z, \theta)$ is a $d_{m}$-vector of functions known up to $\theta$. This is especially so when instruments are used to overcome estimator bias induced by confounding variables, that is, when latent variables causally affect both response and covariates.

Consider the effect of smoking on health. It has been postulated (Leigh and Schembri, 2004) that smoking is related to health through the unobservable confounding variable, risk aversion. Thus cigarette price, being weakly correlated with cigarette consumption and uncorrelated with risk aversion, is a possible but weak instrument. Additional moment inequality information is available here since cigarette consumption and unobserved risk aversion are known to be negatively correlated. A similar scenario arises in the returns to education example of Angrist and Krueger (1991), where quarter of birth is proposed as a (weak) instrument for years of schooling, and schooling and unobserved ability are known to be positively correlated, giving rise to an additional moment inequality condition.

From a technical point of view, progress is still possible in this weak instrument setting provided the strength of the correlation between the instrument and the endogenous regressor is not smaller than $\mu / \sqrt{n}$ for some $\mu \neq 0$ where $n$ is the sample size. For this reason, data are viewed as realisations of the triangular array $\left\{Z_{i n},(i=1, \ldots, n),(n=1,2, \ldots)\right\}$, and any row of the triangular array is endowed with the corresponding expectation operator $\mathbb{E}_{n}$, cf. Example 1 below.

Although moment inequalities taken in isolation typically only have partial or set identifying power, taken together both forms of information can result in a smaller confidence set estimator for $\theta_{0}$ than that based solely on the moment equality constraints. See Manski (2003) and more recently Chernozhukov et al. (2007) and Rosen (2008) for discussions of partial identification. The concern, therefore, of this paper is the construction of a confidence set estimator for $\theta_{0}$ in weakly identified models defined by (1.1) in the presence of additional partially identifying moment inequality (1.2) constraints.

To illustrate the similarities and differences between this paper and the existing literature consider the following example.

\section{Example 1.}

$$
Y_{i}=\theta_{0} X_{i}+\varepsilon_{1 i}, \quad X_{i}=\gamma_{0, n} W_{i}+\vartheta_{0, n} \varepsilon_{1 i}+\varepsilon_{2 i}, \quad i=1, \ldots, n,
$$

where $\varepsilon_{1 i}, \varepsilon_{2 i}$ and $W_{i}$ are mutually uncorrelated. The parameter $\theta_{0}$ is weakly identified if $\gamma_{0, n}=$ $\mathbb{E}_{n}\left[X_{i} W_{i}\right] / \mathbb{E}_{n}\left[W_{i}^{2}\right]=\mu / n^{1 / 2}$ for $\mu \neq 0$, and $\vartheta_{0, n}=\mathbb{E}_{n}\left[X_{i} \varepsilon_{1 i}\right] / \mathbb{E}_{n}\left[\varepsilon_{1 i}^{2}\right]=\vartheta_{0} \neq 0$, and partially identified if $\gamma_{0, n}=0$ and $\vartheta_{0, n}=\vartheta_{0} \geq 0$. Andrews and Soares (2010) considers both non-weak moment equalities and moment inequalities, i.e., $\gamma_{0, n}=\gamma_{0} \neq 0$ and $\vartheta_{0, n}=\vartheta_{0} \geq 0$, while Moon and Schorfheide (2009) considers $\gamma_{0, n}=\gamma_{0} \neq 0$ and $\vartheta_{0, n}=c / n^{1 / 2} \geq 0$, where $c$ is a constant. This paper addresses the case $\gamma_{0, n}=\mu / n^{1 / 2}$ and $\vartheta_{0, n}=\vartheta_{0} \geq 0$.

To aid clarity, the paper focuses on the special case in which no nuisance parameters are present. For recent contributions that discuss inference in partially identified models with nuisance parameters, see Bugni et al. (2015) and Canay and Azeem (2016).

The rest of the paper is organised as follows. Section 2 defines the confidence set estimator for

$\theta_{0}$ and establishes its properties. Section 3 discusses its implementation with Section 4 providing an examination of the finite sample performance of the confidence set estimator. 


\section{Inferential procedure}

Given the sample of observations $\left\{Z_{i n},(i=1, \ldots, n)\right\}$ the interest of the paper is a nominal $\alpha$ level confidence set estimator $\left\{\widehat{C}_{n}(\alpha)\right\}$ for $\theta_{0}$ based on the continuous updating (CUE) generalized method of moments (GMM) estimation criterion (Hansen et al., 1996); cf. Rosen (2008).

Let $\widehat{g}^{n}(\theta)=n^{-1} \sum_{i=1}^{n} g_{i n}(\theta)$ and $\widehat{m}^{n}(\theta)=n^{-1} \sum_{i=1}^{n} m_{i n}(\theta)$ where $g_{i n}(\theta)=g\left(Z_{i n}, \theta\right)$ and $m_{\text {in }}(\theta)=m\left(Z_{\text {in }}, \theta\right),(i=1, \ldots, n)$. The CUE GMM criterion is defined as

$$
\widehat{Q}_{n}(\theta, t)=\left(\begin{array}{c}
\widehat{g}^{n}(\theta) \\
\widehat{m}^{n}(\theta)-t
\end{array}\right)^{\prime} \widehat{V}^{n}(\theta)^{-1}\left(\begin{array}{c}
\widehat{g}^{n}(\theta) \\
\widehat{m}^{n}(\theta)-t
\end{array}\right)
$$

where

$$
\widehat{V}^{n}(\theta)=n^{-1} \sum_{i=1}^{n}\left(\begin{array}{c}
g_{i n}(\theta)-\widehat{g}^{n}(\theta) \\
m_{i n}(\theta)-\widehat{m}^{n}(\theta)
\end{array}\right)\left(\left(g_{i n}(\theta)-\widehat{g}^{n}(\theta)\right)^{\prime},\left(m_{i n}(\theta)-\widehat{m}^{n}(\theta)\right)^{\prime}\right),
$$

and $t \in \mathbb{R}_{+}^{d_{m}}$ is a $d_{m}$-vector of slackness parameters reflecting the inequality moment constraints (1.2). Minimisation with respect to $t$ yields the profile CUE GMM criterion,

$$
\widehat{Q}_{n}(\theta)=\widehat{Q}_{n}\left(\theta, \widehat{t}_{n}(\theta)\right) \quad \text { where } \quad \widehat{t}_{n}(\theta)=\underset{t \in \mathbb{R}_{+}^{d_{m}}}{\operatorname{arginf}} \widehat{Q}_{n}(\theta, t) .
$$

The $\alpha$-level confidence set estimator $\left\{\widehat{C}_{n}(\alpha)\right\}$ based on $(2.1)$ is then defined as

$$
\widehat{C}_{n}(\alpha)=\left\{\theta \in \Theta: n \widehat{Q}_{n}(\theta) \leq q\right\}
$$

where $q$ is a critical value chosen to ensure that $\lim _{n \rightarrow \infty} \operatorname{Pr}_{n}\left(\theta_{0} \in \widehat{C}_{n}(\alpha)\right) \geq 1-\alpha$ and $\operatorname{Pr}_{n}(\cdot)$ is probability taken with respect to the joint distribution of $\left\{Z_{i n}\right\}_{i=1}^{n}$.

Implementation of the confidence set estimator $\widehat{C}_{n}(\alpha)(2.2)$ requires the limit distribution of $\widehat{Q}_{n}\left(\theta_{0}\right)$. Let $\mathbb{E}_{n}[\cdot]$ denote expectation taken with respect to the joint distribution of $\left\{Z_{i n}\right\}_{i=1}^{n}$. Define $g^{n}(\theta)=\mathbb{E}_{n}\left[\widehat{g}^{n}(\theta)\right]$ and $m^{n}(\theta)=\mathbb{E}_{n}\left[\widehat{m}^{n}(\theta)\right]$ likewise. The identified set is then defined by $\Theta_{0}=\cap_{n=1}^{\infty}\left\{\theta \in \Theta: m^{n}(\theta) \geq 0\right\}=\{\theta \in \Theta: m(\theta) \geq 0\}$ where $m(\theta)=\lim _{n \rightarrow \infty} m^{n}(\theta)$; cf. Rosen (2008). The following conditions are imposed.

Condition 1 (Weak Identification).

$$
g^{n}(\theta)=k^{n}(\theta) / n^{1 / 2}
$$

where $\sup _{\theta \in \Theta}\left\|k^{n}(\theta)-k(\theta)\right\|=o(1)$ and $k(\theta)=0$ if and only if $\theta=\theta_{0}$.

Let $\rightsquigarrow$ denote weak convergence of empirical processes. Define the empirical process

$$
\left.\left.\widehat{\Psi}_{n}(\theta)=n^{-1 / 2} \sum_{i=1}^{n}\left(\left(g_{i n}(\theta)\right)-g^{n}(\theta)\right)^{\prime},\left(m_{i n}(\theta)\right)-m^{n}(\theta)\right)^{\prime}\right)^{\prime} .
$$

Condition 2 (Weak Convergence). $\left\{\widehat{\Psi}_{n}(\theta): \theta \in \Theta_{0}\right\} \rightsquigarrow \Psi$, where $\Psi$ is a Gaussian process on $\Theta_{0}$ with mean zero and covariance function $\Delta\left(\theta_{1}, \theta_{2}\right)=\mathbb{E} \Psi\left(\theta_{1}\right) \Psi\left(\theta_{2}\right)^{\prime}$ at $\left(\theta_{1}, \theta_{2}\right), \theta_{1}, \theta_{2} \in \Theta_{0}$. 
Primitive conditions for Condition 2 are given in Theorems 1.5.4 and 1.5.7 of van der Vaart and Wellner (1996) requiring weak convergence of the marginals $\left(\widehat{\Psi}_{n}\left(\theta_{1}\right), \ldots, \widehat{\Psi}_{n}\left(\theta_{k}\right)\right)$ for every finite subset $\theta_{1}, \ldots, \theta_{k}$ of $\Theta_{0}$, stochastic equicontinuity of $\widehat{\Psi}_{n}(\theta)$ and total boundedness of $\Theta_{0}$. For example, Stock and Wright (2000) provide conditions on the data generating process that guarantee Condition 2 is satisfied. In particular, Stock and Wright (2000) require $g_{i n}(\theta)$ and $m_{\text {in }}(\theta)$ to be $m$-dependent sequences for some fixed $m \geq 0$, with the special case $m=0$ corresponding to an independent sequence, and, in addition, to be Lipschitz continuous in expectation over $\Theta_{0}$ with $2+\epsilon$ absolute moments for some $\epsilon>0$ uniformly over $\Theta_{0}$.

Let $V(\theta)=\lim _{n \rightarrow \infty} \operatorname{Var}_{n}\left[n^{1 / 2}\left(\widehat{g}^{n}(\theta)^{\prime}, \widehat{m}^{n}(\theta)^{\prime}\right)^{\prime}\right]$.

Condition 3 (Weight Matrix). The weight matrix $\widehat{V}^{n}(\theta)$ satisfies $\sup _{\theta \in \Theta}\left\|\widehat{V}^{n}(\theta)-V(\theta)\right\|=o_{p}(1)$ where $V(\theta)$ is a non-stochastic strictly positive definite matrix uniformly over $\theta \in \Theta$. Furthermore, $\sup _{\theta \in \Theta}\left\|\widehat{V}^{n}(\theta)\right\|=O_{p}(1)$ for all $n \geq 1$.

Let $b_{\theta}$ denote the number of binding moment conditions at $\theta \in \Theta_{0}$. Also let $b_{0}=b_{\theta_{0}}$. When the context ensures no ambiguity, the dependence of $b_{\theta}$ on $\theta$ is suppressed. Without loss of generality the first $b_{\theta}$ inequality moment conditions are assumed to be binding. Partition $\widehat{m}^{n}(\theta)=\left(\widehat{m}_{b}^{n}(\theta)^{\prime}, \widehat{m}_{c}^{n}(\theta)^{\prime}\right)^{\prime}$ where $\widehat{m}_{b}^{n}(\theta)$ and $\widehat{m}_{c}^{n}(\theta)$ respectively correspond to the binding and non-binding inequality conditions and $c_{\theta}=d_{m}-b_{\theta}$. Define

$$
V_{b}(\theta)=\lim _{n \rightarrow \infty} \operatorname{Var}_{n}\left[n^{1 / 2}\left(\widehat{g}^{n}(\theta)^{\prime}, \widehat{m}_{b}^{n}(\theta)^{\prime}\right)^{\prime}\right] .
$$

Theorem 2.1. Suppose Conditions $1-3$ are satisfied. Then for any constant $C>0$,

$$
\lim _{n \rightarrow \infty} \operatorname{Pr}_{n}\left\{n \widehat{Q}_{n}\left(\theta_{0}\right)>C\right\}=\sum_{j=0}^{b_{0}} w\left(b_{0}, b_{0}-j, V_{b_{0}}\left(\theta_{0}\right)\right) \operatorname{Pr}\left\{\chi_{d_{g}+j}^{2} \geq C\right\}
$$

where $w(\cdot, \cdot, \cdot)$ denotes, mutatis mutandis, the weight function defined in Kudô (1963) and Wolak (1987) and the $\chi_{d_{g}+j}^{2}, j=1, \ldots, b_{0}$, variates are mutually independent.

We emphasise that, though superficially similar, this result is fundamentally different from that in Rosen (2008). The weights $w\left(b_{0}, b_{0}-j, V_{b_{0}}\left(\theta_{0}\right)\right)$ in (2.4) differ from those in Wolak (1987) and Rosen (2008) except in special cases because of the additional presence of the set of weak equality moment conditions (1.1). Details are contained in a supplement available upon request. An explicit expression is only available when $d_{m} \leq 4$; see Rosen (2008). Section 3 provides details of an approximate construction for $\widehat{C}_{n}(\alpha)$.

If the moment inequality conditions (1.2) are omitted from $\widehat{Q}_{n}(\theta)$, then, for any $\theta \notin \Theta_{0}$, the probability that $\theta \in \widehat{C}_{n}(\alpha)$ is positive asymptotically whereas Proposition 3 in Rosen (2008) indicates this probability is 0 asymptotically when binding inequality conditions (1.2) are imposed. Moreover, if only the inequality conditions are imposed $\theta \in \widehat{C}_{n}(\alpha)$ w.p.a.1 for any $\theta \in \Theta_{0}$ such that $m(\theta)>0$. If the weak equality conditions (1.1) are also imposed, then for $\theta \neq \theta_{0}$ but in $\Theta_{0}$ a noncentrality parameter is present due to the presence of $k(\theta)$ which shifts the distribution of $\widehat{Q}_{n}(\theta)$ to the right. As a consequence there is a reduction in the probability that such a value is included in the confidence set. 


\section{Practicalities}

Theorem 2.1 characterises the asymptotic distribution of the scaled criterion function $n \widehat{Q}_{n}(\theta)$ and provides the theoretical basis for the confidence set estimator. However, in practice, it is generally not possible to construct the confidence set exactly since the weights in Theorem 2.1 depend on the parameter $\theta_{0}$ both directly (through $V\left(\theta_{0}\right)$ ) and indirectly (through the number $b_{0}$ of binding constraints). Except in special cases, an explicit expression for the weights is only available when $d_{m} \leq 4$. To deal with the more general setting, two practical procedures to approximate the confidence set estimator are now provided.

Section 4 of Rosen (2008) provides details of a numerical procedure based on Perlman (1969) to obtain critical values. The resulting confidence set is conservative (Rosen, 2008, Corollary 1) but is satisfactory if $b_{0}$ is both small and provides a good approximation to the maximum number of binding inequality moments (Wolak, 1991). Thus, it may sometimes be beneficial to include only a small number of the most effective moment inequalities. While originally proposed for moment inequalities only, the procedure is straightforwardly extended to incorporate weak moment equalities.

The procedure due to Andrews and Soares (2010) selects the set of binding moment inequalities consequently reducing the number of moment inequalities used for inference and, thus, substantially improving the empirical coverage properties of the confidence set estimator. Let

$$
S\left(\left(\begin{array}{c}
g \\
m
\end{array}\right), V\right)=\inf _{t \geq 0}\left(\begin{array}{c}
g \\
m-t
\end{array}\right)^{\prime} V^{-1}\left(\begin{array}{c}
g \\
m-t
\end{array}\right) .
$$

Also let $C$ denote the set of moment selection vectors $\{c\}$, whose $j$ th element $c_{j} \in\{0,1\}$ corresponds to whether or not the $j^{\text {th }}$ moment inequality is selected (see Andrews and Soares, 2010). The estimated moment selection vector $\widehat{c}$ is then obtained as the solution to

$$
\min _{c \in C} S\left(n^{1 / 2}\left(\begin{array}{c}
\widehat{g}^{n}(\theta) \\
c \cdot \widehat{m}^{n}(\theta)
\end{array}\right), \widehat{V}^{n}(\theta)\right)-|c| \sqrt{\log (n)} .
$$

The $d_{m}$-dimensional vector $\varphi$ is constructed according to

$$
\varphi_{j}= \begin{cases}0, & \text { if } \widehat{c}_{j}=1, \\ \infty, & \text { if } \widehat{c}_{j}=0\end{cases}
$$

The critical value is then simulated by the following steps. For each $r=1,2, \ldots, R$, where $R$ is the number of replications, draw $n$ i.i.d. copies of the random vector $Z^{*} \sim N\left(0, I_{d_{g}+d_{m}}\right)$. Then compute $S_{r}=S\left(\widehat{\Omega}^{n}(\theta)^{-1 / 2} Z_{r}^{*}+\left(0_{d_{g}}, \varphi\right), \widehat{\Omega}^{n}(\theta)\right)$ where $0_{d_{g}}$ is zero vector of dimension $d_{g}$,

$$
\widehat{\Omega}^{n}(\theta)=\widehat{D}_{n}^{-1 / 2}(\theta) \widehat{V}_{n}(\theta) \widehat{D}_{n}^{-1 / 2} \text { and } \quad \widehat{D}_{n}(\theta)=\operatorname{diag}\left(\widehat{V}^{n}(\theta)\right) .
$$

Finally the critical value is estimated as

$$
\widehat{q}_{A S}(\theta)=\inf \left\{q: \frac{1}{R} \sum_{r=1}^{R} 1\left\{S_{r} \leq q\right\} \geq 1-\alpha\right\} .
$$




\section{$4 \quad$ Finite sample performance}

All experiments concern the model

$$
Y_{i}=\theta X_{i}+\varepsilon_{i}, \quad X_{i}=\frac{\pi_{1}}{n^{1 / 2}} W_{1 i}+\frac{\pi_{2}}{n^{1 / 2}} W_{2 i}+\nu_{i},(i=1, \ldots, n),
$$

where $\pi_{1}$ and $\pi_{2}$ are constants and $W_{1}$ and $W_{2}$ are each uncorrelated with both error terms $\varepsilon$ and $\nu$ which gives rise to the $d_{g}=2$ weak moment equalities (cf. equation (1.1))

$$
E\left[(Y-\theta X) W_{1}\right]=0 \quad \text { and } \quad E\left[(Y-\theta X) W_{2}\right]=0 .
$$

The additional variables $\left(W_{3}, W_{4}\right)$ are each positively correlated with $\varepsilon$ giving the $d_{m}=2$ moment inequalities (cf. equation (1.2))

$$
E\left[(Y-\theta X) W_{3}\right] \geq 0 \text { and } \quad E\left[(Y-\theta X) W_{4}\right] \geq 0 .
$$

In the simulations, $\left\{W_{1}, W_{2}, W_{3}, W_{4}, \varepsilon, \nu\right\}$ are multivariate normally distributed with all covariance matrix entries zero except $\sigma_{\varepsilon \nu}=\sigma_{\varepsilon w_{3}}=\sigma_{\varepsilon w_{4}}=0.25, \sigma_{\nu w_{3}}=0.5, \sigma_{\nu w_{4}}=-0.5$ and $\sigma_{w_{1}}^{2}=$ $\sigma_{w_{2}}^{2}=\sigma_{\varepsilon}^{2}=\sigma_{\nu}^{2}=\sigma_{w_{3}}^{2}=\sigma_{w_{4}}^{2}=1$. The Supplementary Information provides an additional set of simulations in which the exogenous variables are drawn from the Student $t$ distribution with 5 degrees of freedom yielding a very similar set of results. Thus, in this example, since the identified set $\Theta_{0}=\{\theta \in \Theta: m(\theta) \geq 0\}, \Theta_{0}$ is defined by the moment inequalities $E\left[(Y-\theta X) W_{3}\right] \geq 0$ and $E\left[(Y-\theta X) W_{4}\right] \geq 0$ and equals $[0.5,1.5]$.

\begin{tabular}{ccccccc}
\hline$n$ & $1-\alpha$ & $\mathrm{R}(\mathrm{W} \& \mathrm{P})$ & $\mathrm{AS}(\mathrm{W} \& \mathrm{P})$ & $\mathrm{SW}(\mathrm{W})$ & $\mathrm{K}(\mathrm{W})$ & $\mathrm{R}(\mathrm{P})$ \\
\hline \hline 100 & 0.9 & 1.943 & 2.041 & 2.526 & 2.770 & 2.226 \\
500 & 0.9 & 1.257 & 1.192 & 2.442 & 2.582 & 1.358 \\
1000 & 0.9 & 1.146 & 1.093 & 2.496 & 2.524 & 1.205 \\
\hline 100 & 0.95 & 2.229 & 2.401 & 2.979 & 3.107 & 2.469 \\
500 & 0.95 & 1.372 & 1.316 & 2.934 & 3.036 & 1.424 \\
1000 & 0.95 & 1.217 & 1.167 & 2.955 & 3.030 & 1.251 \\
\hline 100 & 0.99 & 2.821 & 3.154 & 3.488 & 3.608 & 3.023 \\
500 & 0.99 & 1.590 & 1.543 & 3.488 & 3.553 & 1.602 \\
1000 & 0.99 & 1.351 & 1.317 & 3.549 & 3.621 & 1.361 \\
\hline
\end{tabular}

Table 1: Mean width of confidence interval around $\theta_{0}=1$ : $1000 \mathrm{MC}$ replications and $n$ observations.

For each of 1000 Monte Carlo (MC) replications a confidence interval estimator $\widehat{C}_{n}(\alpha)$ is constructed for $\theta=\theta_{0}=1$ based on the Perlman (1969) procedure using both the weak moment equality (cf. equation (4.1)) and the partial moment inequality (cf. equation (4.2)) information. Rosen (2008) also adopts this procedure but using the partial information alone. To distinguish these approaches the notation $\mathrm{R}(\mathrm{P})$ is adopted for the Rosen (2008) procedure and $\mathrm{R}(\mathrm{W} \& \mathrm{P})$ for the extension of Rosen (2008) incorporating the additional weak moment equalities information. Confidence set construction based on the Andrews and Soares (2010) procedure is also considered, adapted to exploit both weak and partial information, and is denotes by $\mathrm{AS}(\mathrm{W} \& \mathrm{P}) . \mathrm{R}(\mathrm{W} \& \mathrm{P})$ and 
$\mathrm{AS}(\mathrm{W} \& \mathrm{P})$ are also compared to constructions based on Stock and Wright (2000) (SW(W)) and Kleibergen (2005) $(\mathrm{K}(\mathrm{W})$ ) based on the weak moment equalities alone.

\begin{tabular}{ccccccc}
\hline $\mathrm{n}$ & $1-\alpha$ & $\mathrm{R}(\mathrm{W} \& \mathrm{P})$ & $\mathrm{AS}(\mathrm{W} \& \mathrm{P})$ & $\mathrm{SW}(\mathrm{W})$ & $\mathrm{K}(\mathrm{W})$ & $\mathrm{R}(\mathrm{P})$ \\
\hline \hline 100 & 0.9 & 0.971 & 0.971 & 0.830 & 0.905 & 1.000 \\
500 & 0.9 & 0.976 & 0.969 & 0.895 & 0.905 & 1.000 \\
1000 & 0.9 & 0.974 & 0.967 & 0.916 & 0.914 & 1.000 \\
\hline 100 & 0.95 & 0.985 & 0.993 & 0.934 & 0.943 & 1.000 \\
500 & 0.95 & 0.985 & 0.981 & 0.946 & 0.951 & 1.000 \\
1000 & 0.95 & 0.984 & 0.978 & 0.942 & 0.957 & 1.000 \\
\hline 100 & 0.99 & 0.997 & 0.998 & 0.983 & 0.982 & 1.000 \\
500 & 0.99 & 0.998 & 0.999 & 0.986 & 0.987 & 1.000 \\
1000 & 0.99 & 0.998 & 0.997 & 0.988 & 0.991 & 1.000 \\
\hline
\end{tabular}

Table 2: $\overline{\text { Coverage probabilities based on } 1000 \text { MC replications, } n \text { observations and } H_{0}: \theta}=\theta_{0}=1$.

Table 1 compares the mean width (over MC replications) of the various confidence interval estimators around $\theta_{0}=1$ with $\pi_{1}=1$ and $\pi_{2}=2$. The third and fourth columns, R(W\&P) and $\mathrm{AS}(\mathrm{W} \& \mathrm{P})$, both use the weak, (4.1), and partial, (4.2), identifying information. Their differences arise because of the different implementations discussed above. The difference between the third and last columns is due solely to the additional weakly identifying information used in the construction $\mathrm{R}(\mathrm{W} \& \mathrm{P})$ over $\mathrm{R}(\mathrm{P})$, the imposition of the additional inequality conditions reducing the width of the confidence intervals appreciably.

Table 2 presents the coverage properties of the various estimators, confirming that those based on our approach, $\mathrm{R}(\mathrm{W} \& \mathrm{P})$ and $\mathrm{AS}(\mathrm{W} \& \mathrm{P})$, are conservative. Note that despite their greater length, the estimators $\mathrm{SW}(\mathrm{W})$ and $\mathrm{K}(\mathrm{W})$ based solely on weakly identifying moment conditions have lower coverage.

Figure 1 plots the empirical power curves for various combinations of $\pi_{1}$ and $\pi_{2}$; viz. $\left\{\pi_{1}=\right.$ $\left.1, \pi_{2}=1\right\},\left\{\pi_{1}=1, \pi_{2}=2\right\},\left\{\pi_{1}=2, \pi_{2}=2\right\}$ and $\left\{\pi_{1}=2, \pi_{2}=3\right\}$. In all cases $\theta=\theta_{0}=1$ and the sample size is $n=1000$. The $\mathrm{SW}(\mathrm{W})$ and $\mathrm{K}(\mathrm{W})$ empirical power curves are much flatter than those of the $\mathrm{AS}(\mathrm{W} \& \mathrm{P}), \mathrm{R}(\mathrm{W} \& \mathrm{P})$ and $\mathrm{R}(\mathrm{P})$ procedures. Although $\mathrm{SW}(\mathrm{W})$ and $\mathrm{K}(\mathrm{W})$ have somewhat higher power inside the identified region, the power of $A S(W \& P), R(W \& P)$ and $R(P)$ is far greater outside the identified region. Since the moment equalities constitute only weakly identifying information, the power of $\mathrm{SW}(\mathrm{W})$ and $\mathrm{K}(\mathrm{W})$ is expected to be relatively low across the whole parameter space. In contrast, the information provided by the moment inequality constraints is partially identifying and, thus, powerful for detecting a false null hypothesis since the true $\theta=\theta_{1}$ then lies outside the identified set, thereby violating the inequalities. Inside the identified region, the moment inequalities are uninformative and, thus, their inclusion effectively adds noise, explaining the lower power of $\mathrm{AS}(\mathrm{W} \& \mathrm{P})$ and $\mathrm{R}(\mathrm{W} \& \mathrm{P})$ as compared with that of $\mathrm{SW}(\mathrm{W})$ and $\mathrm{K}(\mathrm{W})$. The $\mathrm{AS}(\mathrm{W} \& \mathrm{P})$ and $\mathrm{R}(\mathrm{W} \& \mathrm{P})$ approaches dominate $\mathrm{R}(\mathrm{P})$, emphasising the value of the weakly identifying moment information. In particular, as the values of $\pi_{1}$ and $\pi_{2}$ increase, the $\mathrm{AS}(\mathrm{W} \& \mathrm{P})$ and $\mathrm{R}(\mathrm{W} \& \mathrm{P})$ power curves lie significantly above that of $\mathrm{R}(\mathrm{P})$. 

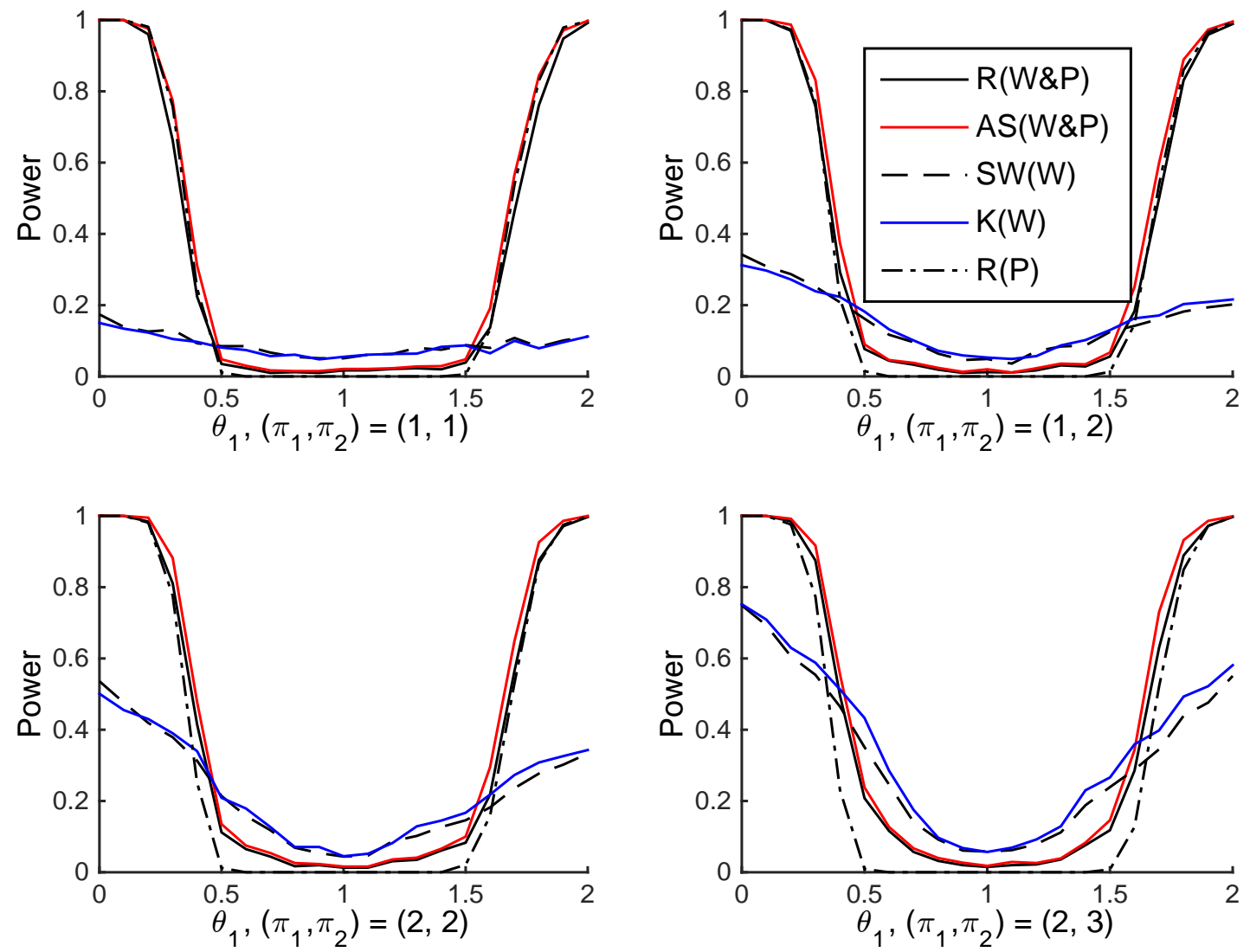

Figure 1: Estimated power against the alternative $\theta=\theta_{1} \in[0,2]$ for $\theta_{0}=1, n=1000$ and various values of $\pi_{1}$ and $\pi_{2}$.

\section{$5 \quad$ Proof of Theorem 2.1}

Let

$$
\bar{v}^{n}(\theta)=n^{1 / 2}\left(\begin{array}{c}
\widehat{g}^{n}(\theta)-g^{n}(\theta) \\
\widehat{m}^{n}(\theta)-m^{n}(\theta)
\end{array}\right), \quad \bar{v}_{b}^{n}(\theta)=n^{1 / 2}\left(\begin{array}{c}
\widehat{g}^{n}(\theta)-g^{n}(\theta) \\
\widehat{m}_{b}^{n}(\theta)-m_{b}^{n}(\theta)
\end{array}\right) .
$$

where $m^{n}(\theta)=\left(m_{b}^{n}(\theta)^{\prime}, m_{c}^{n}(\theta)^{\prime}\right)^{\prime}$ is partitioned conformably with $\widehat{m}^{n}(\theta)$. By Condition 1 ,

$$
\begin{aligned}
n \widehat{Q}_{n}(\theta) & =\min _{t \geq 0}\left(\bar{v}^{n}(\theta)+\left(\begin{array}{c}
n^{1 / 2} g^{n}(\theta) \\
n^{1 / 2} m^{n}(\theta)-t
\end{array}\right)\right)^{\prime} \widehat{V}^{n}(\theta)^{-1}\left(\bar{v}^{n}(\theta)+\left(\begin{array}{c}
n^{1 / 2} g^{n}(\theta) \\
n^{1 / 2} m^{n}(\theta)-t
\end{array}\right)\right) \\
& =\min _{s \geq-n^{1 / 2} m^{n}(\theta)}\left(\bar{v}^{n}(\theta)-\left(\begin{array}{c}
-k^{n}(\theta) \\
s
\end{array}\right)\right)^{\prime} \widehat{V}^{n}(\theta)^{-1}\left(\bar{v}^{n}(\theta)-\left(\begin{array}{c}
-k^{n}(\theta) \\
s
\end{array}\right)\right) \\
& =\min _{s_{b} \geq 0, s_{c} \geq-n^{1 / 2} m_{c}^{n}(\theta)}\left(\bar{v}^{n}(\theta)-\left(\begin{array}{c}
-k^{n}(\theta) \\
s
\end{array}\right)\right)^{\prime} \widehat{V}^{n}(\theta)^{-1}\left(\bar{v}^{n}(\theta)-\left(\begin{array}{c}
-k^{n}(\theta) \\
s
\end{array}\right)\right),
\end{aligned}
$$


where $s=\left(s_{b}^{\prime}, s_{c}^{\prime}\right)^{\prime}$ is partitioned conformably with $m^{n}(\theta)$. Since $m_{c}^{n}(\theta)>0, n^{1 / 2} m_{c}^{n}(\theta) \rightarrow \infty$ and thus, invoking also Condition 3 ,

$$
n \widehat{Q}_{n}(\theta)-\min _{s_{b} \geq 0, s_{c} \in \mathbb{R}^{c}}\left(\bar{v}^{n}(\theta)-\left(\begin{array}{c}
-k(\theta) \\
s
\end{array}\right)\right)^{\prime} V(\theta)^{-1}\left(\bar{v}^{n}(\theta)-\left(\begin{array}{c}
-k(\theta) \\
s
\end{array}\right)\right)=o_{p}(1) .
$$

By Lemma 1 of Rosen (2008)

$$
\begin{aligned}
& \min _{s_{b} \in \mathbb{R}_{+}^{b}, s_{c} \in \mathbb{R}^{c}}\left(\bar{v}^{n}(\theta)-\left(\begin{array}{c}
-k(\theta) \\
s
\end{array}\right)\right)^{\prime} V(\theta)^{-1}\left(\bar{v}_{n}(\theta)-\left(\begin{array}{c}
-k(\theta) \\
s
\end{array}\right)\right) \\
= & \min _{s_{b} \in \mathbb{R}_{+}^{b}}\left(\bar{v}_{b}^{n}(\theta)-\left(\begin{array}{c}
-k(\theta) \\
s_{b}
\end{array}\right)\right)^{\prime} V_{b}(\theta)^{-1}\left(\bar{v}_{b}^{n}(\theta)-\left(\begin{array}{c}
-k(\theta) \\
s_{b}
\end{array}\right)\right) .
\end{aligned}
$$

By Condition 2, $\bar{v}_{b}^{n} \rightsquigarrow \bar{v}_{b}$ where $\bar{v}_{b}$ is a zero mean Gaussian process on $\Theta_{0}$, thus, for all $\theta \in \Theta_{0}$ $\bar{v}_{b}(\theta) \sim N\left(0, V_{b}(\theta)\right)$. It follows that, for any $\theta \in \Theta_{0}$,

$$
n \widehat{Q}_{n}(\theta) \rightarrow_{d} \min _{s_{b} \in \mathbb{R}_{+}^{b}}\left(\bar{v}_{b}-\left(\begin{array}{c}
-k(\theta) \\
s_{b}
\end{array}\right)\right)^{\prime} V_{b}(\theta)^{-1}\left(\bar{v}_{b}-\left(\begin{array}{c}
-k(\theta) \\
s_{b}
\end{array}\right)\right)=Q^{b}(\theta),
$$

and using the results of Wolak (1991) based on Kudô (1963),

$$
\operatorname{Pr}\left\{Q^{b}\left(\theta_{0}\right) \geq C\right\}=\sum_{j=0}^{b} w\left(b, b-j, V_{b}\left(\theta_{0}\right)\right) \operatorname{Pr}\left\{\chi_{d_{g}+j}^{2} \geq C\right\}
$$

\section{Acknowledgements}

The authors are grateful to the anonymous referees for helpful feedback. Feng thanks the National Science Foundation of China (grant 71301026) for financial support.

\section{References}

Andrews, D. W. K. and Cheng, X. (2012). Estimation and inference with weak, semi-strong, and strong identification. Econometrica $802153-2211$.

Andrews, D. W. K. and SoAres, G. (2010). Inference for parameters defined by moment inequalities using generalized moment selection. Econometrica 78 119-157.

Angrist, J. and Krueger, A. (1991). Does compulsory school attendence affect schooling and earnings. Quart. J. Econ. 106 993-1014.

Bugni, F., Canay, I. and Shi, X. (2015). Inference for functions of partially identified parameters in moment inequality models. Cemmap working paper 10.1920/wp.cem.2015.5415 . 
Canay, I. and Azeem, S. (2016). Practical and theoretical advances in inference for partially identified models. Cemmap working paper 051616 .

Chernozhukov, V., Hong, H. and Tamer, E. (2007). Estimation and confidence regions for parameter sets in econometric models. Econometrica 75 1243-1284.

Guggenberger, P. and Smith, R. J. (2005). Generalized empirical likelihood estimators and tests under partial, weak, and strong identification. Econometric Theory 21 667-709.

Hansen, L. P., Heaton, J. and Amir, Y. (1996). Finite-sample properties of some alternative gmm estimators. Journal of Business and Economic Statistics 14 262-280.

Imbens, G. W. (2014). Instrumental variables: an econometrician's perspective. Statist. Sci. 29 $323-358$.

Kleibergen, F. (2005). Testing parameters in GMM without assuming that they are identified. Econometrica 73 1103-1123.

Kudô, A. (1963). A multivariate analogue of the one-sided test. Biometrika 50 403-418.

Leigh, J. and Schembri, M. (2004). Instrumental variables technique: cigarette price provided better estimate of effects of smoking on sf-12. Journal of clinical epidemiology 57 284-293.

Lipsitz, S. R. and Ibrahim, J. G. (1998). Estimating equations with incomplete categorical covariates in the Cox model. Biometrics 54 1002-1013.

Manski, C. (2003). Partial Identification of Probability Distributions. Springer-Verlag, New York.

Moon, H. R. and Schorfheide, F. (2009). Estimation with overidentifying inequality moment conditions. J. Econometrics 153 136-154.

Perlman, M. D. (1969). One-sided testing problems in multivariate analysis. Ann. Math. Statist. $40549-567$.

Rosen, A. M. (2008). Confidence sets for partially identified parameters that satisfy a finite number of moment inequalities. J. Econometrics 146 107-117.

ShPITSER, I. (2014). Causal graphs: addressing the confounding problem without instruments or ignorability. Statist. Sci. 29 367-370.

Stock, J. H. and Wright, J. H. (2000). GMM with weak identification. Econometrica 68 1055-1096.

VAn Der VAart, A. W. (1998). Asymptotic statistics, vol. 3 of Cambridge Series in Statistical and Probabilistic Mathematics. Cambridge University Press, Cambridge.

VAn Der VAart, A. W. and Wellner, J. A. (1996). Weak convergence and empirical processes. Springer Series in Statistics, Springer-Verlag, New York. With applications to statistics. 
Wolak, F. A. (1987). An exact test for multiple inequality and equality constraints in the linear regression model. J. Amer. Statist. Assoc. 82 782-793.

Wolak, F. A. (1991). The local nature of hypothesis tests involving inequality constraints in nonlinear models. Econometrica 59 981-995. 